\title{
O manejo da síndrome do desconforto respiratório agudo
}

\author{
Management of the acute respiratory distress syndrome
}

\author{
Alexandre Tellechea Rotta ${ }^{1}$, Cláudia Laura Barberio Kunrath ${ }^{2}$, Budi Wiryawan ${ }^{3}$
}

\section{Resumo}

Objetivo: Revisar as atuais estratégias de suporte e de tratamento da síndrome do desconforto respiratório agudo (SDRA).

Fontes dos dados: Dados próprios de nosso laboratório de pesquisa e bibliografia relacionada às áreas de SDRA e lesão pulmonar aguda, pesquisados através do Medline.

Síntese dos dados: Apesar de avanços no entendimento da sua patogênese, a SDRA ainda resulta em significativa morbidade e mortalidade. A ventilação mecânica é a principal modalidade terapêutica na SDRA, sendo atualmente considerada não mais apenas uma medida de suporte, mas sim uma terapia capaz de alterar o curso da patologia. Novas estratégias ventilatórias, como a ventilação oscilatória de alta frequiência (VOAF), têm-se mostrado promissoras. Neste texto, revisamos o conhecimento atual no manejo da SDRA, incluindo ventilação mecânica convencional e não convencional, uso de surfactante, óxido nítrico, moduladores do processo inflamatório, oxigenação extracorpórea e posição prona.

Conclusões: A última década foi marcada por avanços significativos, como o conceito de ventilação mecânica protetora na SDRA. O benefício da aplicação de estratégias alternativas, como a VOAF, assim como do uso do surfactante exógeno e moduladores de inflamação continuam sendo alvo de estudo.

J Pediatr (Rio J) 2003;79(Supl.2):S149-S160: Síndrome do desconforto respiratório agudo, ventilação mecânica, ventilação de alta freqüência, surfactante, óxido nítrico.
1. Professor assistente de Pediatria e Medicina de Emergência, State University of New York at Buffalo, Diretor Associado da Unidade de Terapia Intensiva Pediátrica. The Children's Hospital of Buffalo. Buffalo, New York, USA.

2. Médica residente do Departamento de Pediatria. The Children's Hospital of Buffalo. Buffalo, New York, USA.

3. Fellow em Terapia Intensiva Pediátrica. The Children's Hospital of Buffalo. Buffalo, New York, USA.

\begin{abstract}
Objective: To review the current support and treatment strategies of the Acute Respiratory Distress Syndrome (ARDS)

Data sources: Original data from our research laboratory and from representative scientific articles on ARDS and acute lung Injury searched through Medline.

Summary of the findings: Despite advances in the understanding of the pathogenesis of ARDS, this syndrome still results in significant morbidity and mortality. Mechanical ventilation, the main therapeutic modality for ARDS, is no longer considered simply a support modality, but a therapy capable of influencing the course of the disease. New ventilation strategies, such as high-frequency oscillatory ventilation (HFOV) appear to be promising. This text reviews the current knowledge of ARDS management, including conventional and non-conventional ventilation, the use of surfactant, nitric oxide, modulators of inflammation, extracorporeal membrane oxygenation and prone position.
\end{abstract}

Conclusions: The last decade was marked by significant advances, such as the concept of protective ventilation for ARDS. The benefit of alternative strategies, such as HFOV, the use of surfactant and immunomodulators continue to be the target of study.

JPediatr(Rio J) 2003;79(Supl.2):S149-S160: Acute respiratory distress syndrome, mechanical ventilation, high-frequency ventilation, surfactant, nitric oxide.

\section{Introdução}

A síndrome do desconforto respiratório agudo (SDRA) é uma entidade marcada por significativa resposta inflamatória a um insulto local (pulmonar) ou distante (sistêmico) que resulta, invariavelmente, em hipoxemia e marcada alteração da mecânica pulmonar. Por definição, quatro 
critérios clínicos devem estar presentes para que se estabeleça o diagnóstico de SDRA ${ }^{1}$ : (1) doença de acometimento agudo, (2) infiltrados pulmonares bilaterais na radiografia de tórax, (3) pressão capilar da artéria pulmonar $\leq 18 \mathrm{~mm}$ $\mathrm{Hg}$ ou ausência de evidência clínica de hipertensão atrial esquerda, e (4) razão entre a pressão parcial arterial de oxigênio $\left(\mathrm{PaO}_{2}\right)$ e a fração inspirada de oxigênio $\left(\mathrm{FiO}_{2}\right)$ $\leq 200$. Pacientes que preenchem critérios 1 a 3 , mas apresentam uma razão $\mathrm{PaO}_{2} / \mathrm{FiO}_{2}>200 \mathrm{e} \leq 300$, são considerados como tendo lesão pulmonar aguda (LPA), um processo patofisiológico semelhante à SDRA, mas de menor severidade clínica. Usando os critérios acima, estima-se que a SDRA tenha uma incidência de 13,5 casos por 100.000 pessoas, e que a LPA acometa 17,9 a cada 100.000 pessoas ${ }^{2}$. Mesmo com significativos avanços nos cuidados gerais de terapia intensiva, as dramáticas alterações características da SDRA estão associadas a uma elevada mortalidade, variando entre $35 \%$ e $71 \%$.

Apesar de ter sido descrita há várias décadas ${ }^{6}$ e ser uma causa importante de morbimortalidade em unidades de terapia intensiva pediátrica em todo o mundo, a SDRA não dispõe de um tratamento farmacológico específico. Entretanto, avanços no entendimento da patogênese e fisiopatologia da SDRA, ao longo dos anos, proporcionaram a evolução de uma série de terapias de suporte capazes de causar impacto no desfecho de pacientes acometidos por esta patologia (Tabela 1).

Tabela 1 - Estratégias terapêuticas na SDRA

Controle do fator causador (sepse, choque, etc.)
Ventilação mecânica
- Exposição controlada ao oxigênio
- Prevenção de volutrauma (uso de volume corrente reduzido)
- Prevenção de atelectrauma (uso de PEEP adequada)
Administração criteriosa de fluidos
Otimização hemodinâmica e do aporte de oxigênio tecidual
Ventilação não convencional
- Ventilação de alta frequiência
- Ventilação não invasiva
- Ventilação líquida
Terapia medicamentosa
- Surfactante
- Óxido nítrico
- Corticosteróides e outros antiinflamatórios
Oxigenação de membrana extracorpórea (OMEC)
Terapia de posição (prona)
Prevenção e diagnóstico precoce de infecções intercorrentes
Analgesia e sedação
Suporte nutricional
Suporte psicológico (paciente e familiares)

Apesar de apresentarem sucesso no ambiente experimental do laboratório, muitas das modalidades disponíveis para o manejo da SDRA não se mostraram eficazes, ou ainda não foram propriamente testadas na prática clínica. Isto deve-se, primariamente, ao fato de que pacientes com SDRA formam uma população extremamente heterogênea, que necessita ser avaliada em estudos com grandes amostras, consumindo recursos significativos e exigindo grande capacidade de integração entre os centros participantes. A disponibilidade de dados clínicos específicos para a população pediátrica com SDRA é ainda mais limitada, devido à quase inexistência de estudos controlados nesta população. Assim sendo, muitas das estratégias utilizadas no manejo da SDRA pediátrica, bem como suas indicações, foram adaptadas ou inferidas de estudos com pacientes adultos.

\section{Estratégias de tratamento da SDRA Controle do fator causal}

Apesar da SDRA não ter tratamento específico, muitos dos fatores que causam e perpetuam esta patologia podem ser tratados ou controlados. Por exemplo, pacientes com choque hipovolêmico devem ser prontamente identificados e tratados com rápida reposição volumétrica, de forma a minimizar o impacto deste estado na evolução e manutenção da SDRA. Da mesma forma, pacientes com abdômen agudo infeccioso devem ser tratados com antibióticos e intervenção cirúrgica precoce, quando indicada. Pacientes com choque séptico ou pneumonias que evoluem para SDRA devem ser prontamente tratados com expansão intravascular e antibióticos, uma vez que o tratamento do fator causador infeccioso e controle hemodinâmico são fundamentais no sucesso do manejo da patologia pulmonar subseqüente.

\section{Exposição controlada ao oxigênio}

Pacientes com SDRA apresentam, por definição, hipoxemia significativa (razão $\left.\mathrm{PaO}_{2} / \mathrm{FiO}_{2}<200\right)^{1}$. Por este motivo, a administração de oxigênio é indicada no manejo da fase inicial da insuficiência respiratória aguda. A severa hipoxemia em pacientes com SDRA deve-se ao curtocircuito intrapulmonar, onde zonas não ventiladas em decorrência de edema, atelectasia ou consolidação continuam a receber aporte sangüíneo, apesar de serem incapazes de participar na oxigenação do mesmo. A oxigenoterapia por via de máscara, tenda ou mesmo por equipamento de ventilação não invasiva é capaz de causar uma melhora sintomática no paciente em fase inicial de falência respiratória aguda. Entretanto, a rápida progressão natural da SDRA com diminuição da complacência pulmonar, o aumento do trabalho dos músculos respiratórios e a subseqüente exaustão, fazem com que a oxigenoterapia tenha valor apenas como medida de alívio sintomático temporário, enquanto prepara-se a instituição de ventilação pulmo- 
nar mecânica. A maioria de pacientes com critérios diagnósticos de SDRA não pode ser manejada exclusivamente com oxigenoterapia, necessitando de ventilação mecânica. O profissional da saúde que entende o processo fisiopatológico da SDRA deve reconhecer que o paciente com critérios diagnósticos estabelecidos, e que apresenta acelerado aumento da necessidade de oxigênio, necessita de ventilação mecânica. A oxigenoterapia não deve retardar a instituição do suporte ventilatório, uma vez que a intubação e a iniciação da ventilação mecânica na SDRA devem ser estabelecidas de forma eletiva, antes do paciente apresentar franca falência respiratória.

A administração de oxigênio, apesar de simples, não é livre de efeitos adversos. A exposição continuada a altas concentrações de oxigênio $\left(\mathrm{FiO}_{2} \geq 0,6\right)$ é capaz de causar dano pulmonar, mesmo na ausência de lesão préexistente $^{7}$. O dano pulmonar resultante da toxicidade pelo oxigênio é decorrente de radicais livres e de substâncias reativas de oxigênio, gerados espontaneamente em ambientes hiperóxicos ou através de produtos da ativação de neutrófilos e macrófagos alveolares ${ }^{8,9}$. O pulmão normal lida com insultos oxidativos através de uma série de enzimas (superóxido dismutase, glutation peroxidase, glutation redutase, catalase) ou antioxidantes (vitaminas C e E, albumina, etc.), sendo capaz de tolerar elevadas concentrações de oxigênio por vários dias. Entretanto, o pulmão lesado e exposto a concentrações moderadas de oxigênio (não nocivas a pulmões normais) pode causar piora do dano tecidual pulmonar, mesmo quando a exposição ao oxigênio é limitada a apenas algumas horas ${ }^{8}$. Este fenômeno ocorre, presumivelmente, devido ao desequilíbrio entre estímulos oxidativos e mecanismos de proteção antioxidantes encontrados em estados de lesão pulmonar aguda.

\section{Ventilação mecânica}

A ventilação pulmonar mecânica continua sendo a principal modalidade de suporte na SDRA e é indicada na maioria dos $\operatorname{casos}^{10}$. Apesar disso, as indicações para instituição da ventilação mecânica em pacientes com SDRA são, de certa forma, vagas, baseadas em um conjunto de dados clínicos (dispnéia, taquipnéia, uso e fadiga de musculatura acessória, sudorese, má perfusão, etc.), laboratoriais (acidose, hipoxemia, hipercapnia) e radiológicos (piora do infiltrado alveolar). Uma tentativa de objetivar critérios para a instituição de ventilação mecânica na SDRA é a chamada "regra dos 50", onde uma $\mathrm{PaO}_{2}<50$ torr e uma $\mathrm{PaCO}_{2}>50$ torr com uma $\mathrm{FiO}_{2}$ de $50 \%$ caracterizaria pacientes que necessitam de suporte ventilatório. Estes critérios, entretanto, identificam pacientes bastante graves e com falência respiratória iminente. Um dos pontos chave no tratamento da SDRA é a identificação precoce dos pacientes com comprometimento respiratório, de forma que a ventilação mecânica seja iniciada antes que estes desenvolvam um estado extremo de falência respiratória.
A heterogeneidade da distribuição da doença pulmonar em pacientes com SDRA torna a ventilação mecânica um desafio para o intensivista. Na SDRA típica, áreas pulmonares dependentes da gravidade apresentam denso infiltrado inflamatório alveolar e intersticial, edema, detritos celulares, atelectasia e consolidação, enquanto que as áreas não dependentes são relativamente poupadas (Figura 1) ${ }^{11}$. No pulmão sadio com tensão superficial homogênea, o volume corrente se dispersa de maneira equilibrada entre diferentes segmentos pulmonares.
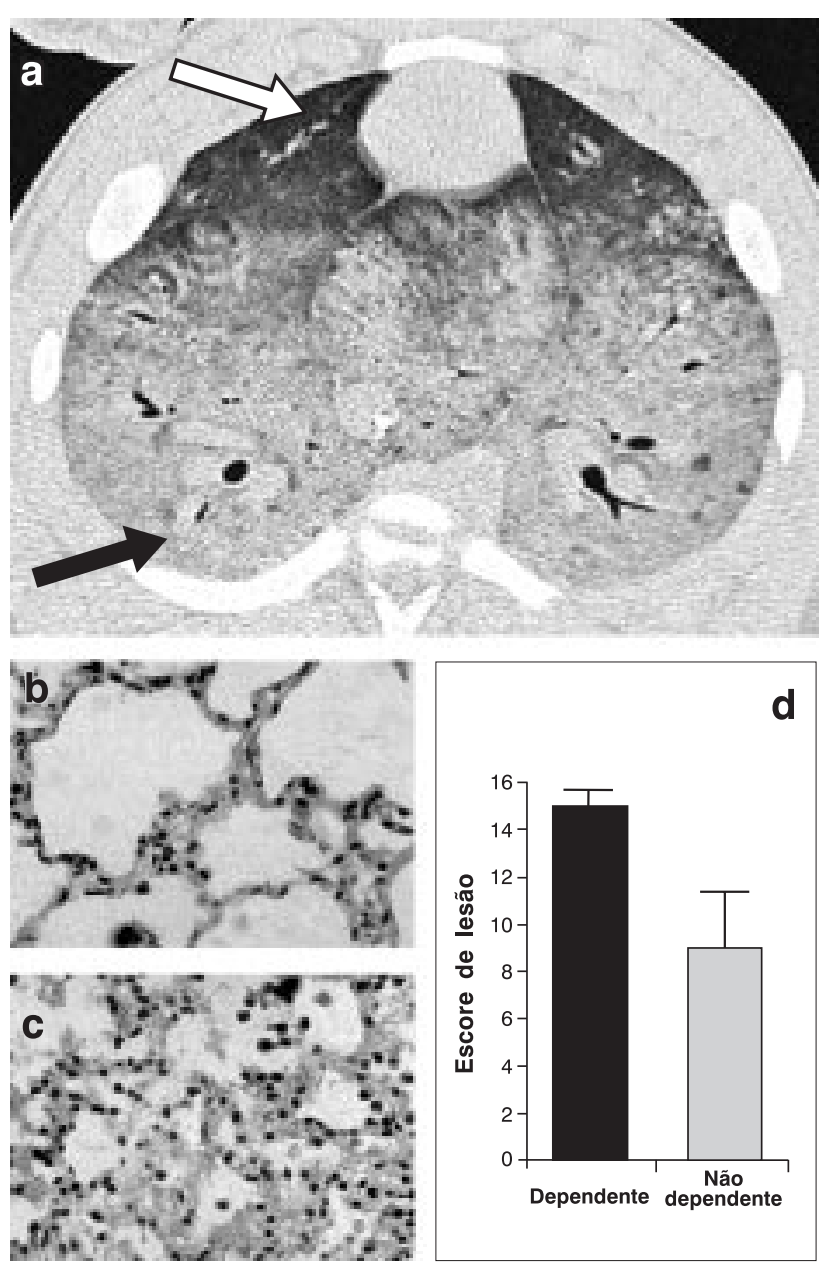

Figura 1 - a) Tomografia axial computadorizada de um modelo experimental (suíno) de SDRA mostrando a distribuição heterogênea da patologia pulmonar. A região pulmonar não dependente da gravidade (seta branca) tem aspecto relativamente normal, enquanto que a região pulmonar dependente (seta preta) apresenta maior envolvimento. A análise histológica de mostras de outro modelo animal de SDRA (coelho) também demonstra a distribuição heterogênea desta patologia, com menor evidência de inflamação e dano tecidual na região nãodependente (b), e contraste com a região dependente (c). A aplicação de um escore histopatológico pulmonar objetivo (d), neste mesmo modelo, confirma a distribuição heterogênea da lesão tecidual 
Já em pacientes com SDRA, o volume corrente segue a via de menor impedimento, com tendência a distender exageradamente os alvéolos mais complacentes (não dependentes) enquanto que falha em recrutar os alvéolos menos complacentes nas áreas dependentes. Além de heterogênea, a doença pulmonar na SDRA é dinâmica ${ }^{12}$, e áreas de complacência relativamente adequadas podem adquirir características de complacência bastante diminuídas em questão de horas, acompanhando a rápida evolução desta síndrome.

A ventilação mecânica na SDRA é muito mais do que uma mera terapia de suporte utilizada para dar tempo à resolução da doença pulmonar. Hoje, sabemos que a estratégia ventilatória empregada é capaz de influenciar a progressão da doença pulmonar, podendo resultar em desfechos mais favoráveis quando estratégias protetoras são utilizadas. Da mesma forma, o uso de estratégias não protetoras está relacionado a desfechos fisiológicos menos favoráveis e a uma elevada mortalidade ${ }^{5,13-15}$.

\section{Volume corrente $(V C)$}

A utilização de VC inadequadamente alto em modelos experimentais é capaz de causar dano pulmonar, mesmo em pulmões normais ${ }^{16}$. Em modelos de SDRA, um VC historicamente considerado adequado, como 10 $\mathrm{ml} / \mathrm{kg}$, está associado à progressão e piora da lesão pulmonar ${ }^{17}$. Isto ocorre porque, em estados de baixa complacência pulmonar, a introdução de um VC moderado ou alto pode levar à distensão exagerada de alvéolos, marcada pelo ponto de inflexão superior da relação entre volume e pressão estática de via aérea (Figura 2), resultando no chamado "volutrauma". Usando este prin-

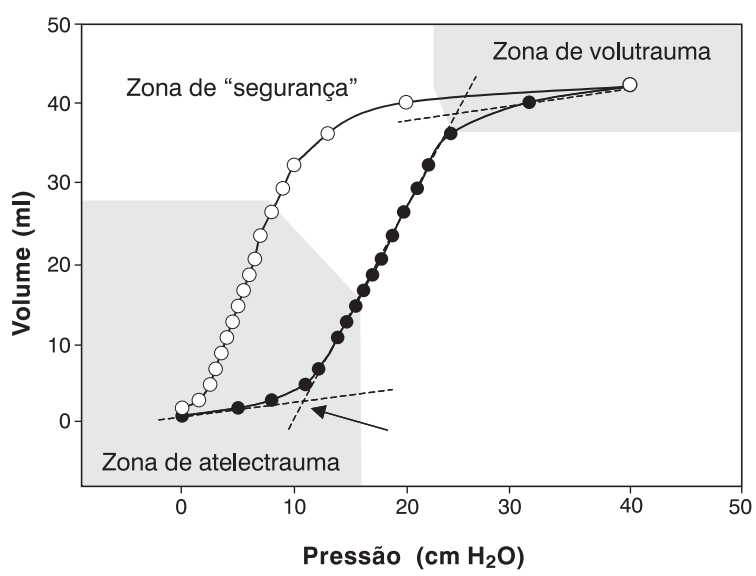

Figura 2 - Curva estática da relação entre pressão e volume do sistema respiratório em um modelo animal de SDRA (coelho). A seta indica o ponto de inflexão inferior cípio, Amato e colaboradores ${ }^{5}$ demonstraram uma significativa redução da mortalidade aos 28 dias, em pacientes com SDRA tratados com uma estratégia de pulmão aberto, que incluía $\mathrm{VC}$ menor que $6 \mathrm{ml} / \mathrm{kg}$ e PEEP acima do ponto de inflexão inferior. Entretanto dois outros estudos, empregando apenas VC reduzido ${ }^{18,19}$, falharam em mostrar benefício desta estratégia em pacientes com SDRA. Mais recentemente, um estudo multicêntrico norte-americano, envolvendo 861 pacientes com SDRA $^{15}$, mostrou uma redução de $22 \%$ na mortalidade de pacientes tratados com $\mathrm{VC}$ reduzido $(6 \mathrm{ml} / \mathrm{kg})$ em comparação com VC tradicional $(12 \mathrm{ml} / \mathrm{kg}$ ). A discrepância nos resultados dos estudos multicêntricos está relacionada a diversidades metodológicas importantes, como os diferentes valores de VC empregados nos grupos de intervenção e controle (Figura 3). Apenas os estudos com uma adequada diferença de $\mathrm{VC}$ entre o grupo de volume reduzido e o grupo controle ${ }^{5,15}$ apresentaram resultados positivos.

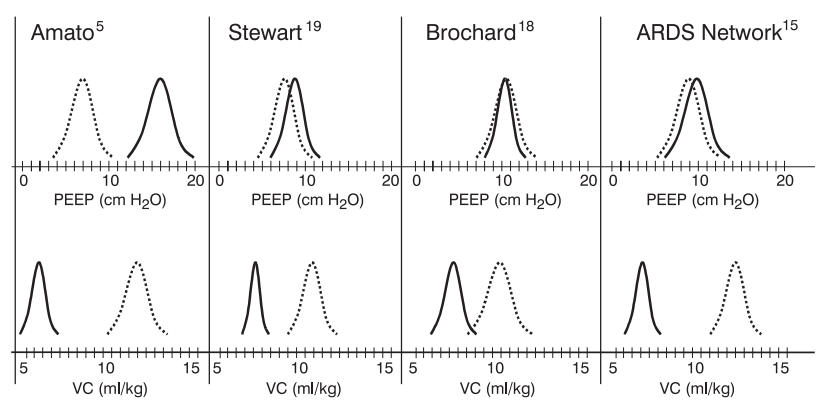

Figura 3 - Comparação da PEEP e volume corrente (VC) entre diferentes estudos randomizados e controlados utilizando uma estratégia de VC reduzido na SDRA

Não existem, até o momento, estudos clínicos testando a hipótese de que o uso de VC reduzido resultaria em benefício na população pediátrica. Entretanto, considerando que a recomendação do uso de $\mathrm{VC}$ reduzido tem forte embasamento fisiológico, experimental e clínico (em adultos), pacientes pediátricos com SDRA devem receber ventilação mecânica com um $\mathrm{VC}$ igual ou menor que $6 \mathrm{ml} / \mathrm{kg}$ até que dados específicos para esta população sejam disponíveis.

\section{Pressão expiratória final positiva (PEEP)}

Na SDRA, alvéolos da região pulmonar dependente da gravidade apresentam complacência exageradamente reduzida em relação a alvéolos não dependentes. Assim sendo, a cada expiração, os alvéolos dependentes atingem um volume crítico de fechamento, resultando no colabamento alveolar. Em seqüência, durante a inspiração, estes 
alvéolos colabados são reabertos. A repetição cíclica de abertura e colabamento alveolar gera forças bastante altas e capazes de causar lesão tecidual (atelectrauma). O uso da PEEP tem como objetivo principal evitar que alvéolos menos complacentes colapsem quando do término da expiração. O uso excessivo da PEEP aumenta o risco de pneumotórax, gera hiperinsuflação de certos segmentos pulmonares e pode causar efeitos adversos hemodinâmicos, por aumento da pressão intratorácica e diminuição do retorno venoso (pré-carga). Entretanto, o uso da PEEP inadequadamente baixa durante a ventilação mecânica provoca o colabamento e a reabertura alveolar de forma cíclica e resulta em atelectrauma.

O uso da PEEP com nível adequado para manter o volume pulmonar durante a expiração está associado a desfechos fisiológicos favoráveis ${ }^{13,16,17}$. Como já mencionado acima, Amato e colaboradores ${ }^{5}$ demonstraram uma redução da mortalidade aos 28 dias em pacientes ventilados com VC menor que $6 \mathrm{ml} / \mathrm{kg}$ e PEEP aplicado acima do ponto de inflexão inferior. É impossível discernir se os efeitos observados ${ }^{5}$ são atribuíveis à limitação do VC, ao uso de PEEP adequado ou a ambos (Figura 3). Entretanto, estratégias que aplicam PEEP adequado ao mesmo tempo que evitam super-distensão alveolar são capazes de prevenir a geração de substâncias pró-inflamatórias (biotrauma) que podem adversamente afetar a seqüência de progressão da lesão pulmonar ${ }^{17,20}$, bem como causar dano a órgãos distantes pela entrada dessas substâncias na circulação ${ }^{21}$. Apesar do papel protetor da PEEP ter sido sistematicamente documentado em estudos de laboratório, o estudo clínico multicêntrico norteamericano de pacientes tratados com volume pulmonar expiratório alto e $\mathrm{FiO}_{2}$ baixo, comparados com pacientes tratados com volume pulmonar expiratório baixo e $\mathrm{FiO}_{2}$ alto, foi recentemente terminado por futilidade após a inclusão de 550 pacientes $^{22}$.

Na prática clínica, pacientes pediátricos com SDRA devem ser ventilados com PEEP capaz de manter um volume pulmonar adequado no final da expiração. Este valor é geralmente maior que $8 \mathrm{~cm} \mathrm{H}_{2} \mathrm{O}$ e menor que $20 \mathrm{~cm}$ $\mathrm{H}_{2} \mathrm{O}$, salvo extraordinárias exceções. A PEEP deve ser aumentada progressivamente (em alíquotas de 2 a $3 \mathrm{~cm}$ $\mathrm{H}_{2} \mathrm{O}$ ), para otimizar a oxigenação (saturação entre 90 e $95 \%$ com $\left.\mathrm{FiO}_{2} \leq 0,5\right)$, e a insuflação pulmonar, checada na radiografia de tórax ou tomografia computadorizada. Pacientes com anasarca severa ou outras lesões restritivas torácicas (queimaduras circunferenciais), bem como pacientes com pressão abdominal excessiva podem necessitar de PEEPs mais elevadas.

Uma estratégia que limita o $\mathrm{VC}$ e ao mesmo tempo aplica uma PEEP ideal, geralmente resulta em redução do volume minuto com conseqüente hipercapnia, mesmo com aumento da frequiência respiratória ${ }^{17}$. Estratégias com hipercapnia permissiva (hipoventilação controlada), em que a elevação de $\mathrm{PaCO}_{2}$ até aproximadamente 80 torr é aceita, desde que mantendo o pH acima de 7,25, são bem toleradas em adultos ${ }^{23}$, mas podem ter efeitos adversos ${ }^{24} \mathrm{e}$ não foram testadas adequadamente em crianças. Na nossa prática clínica, pacientes pediátricos com SDRA, que desenvolvem hipercapnia significativa quando da instituição de ventilação protetora (PEEP elevado com VC limitado), são prontamente colocados em ventilação oscilatória de alta freqüência.

\section{Modo de ventilação}

Ventiladores mecânicos convencionais modernos oferecem uma crescente variedade de modos de ventilação para o paciente com SDRA. Entretanto, conceptualmente, a maioria dos modos de ventilação na SDRA se assemelham pelo fato de que são ciclados por tempo e limitados por volume ou pressão. Um modo ciclado por tempo e limitado por volume significa que o ciclo (inspiração e expiração) é controlado por tempo (tempo inspiratório e frequiência respiratória), sendo que, durante a fase inspiratória do ciclo, um certo volume pré-determinado pelo operador é administrado. Um modo ciclado por tempo e limitado por pressão significa que o ciclo (inspiração e expiração) é controlado por tempo (tempo inspiratório e frequiência respiratória), sendo que, durante a fase inspiratória do ciclo, uma certa pressão pré-determinada pelo operador é administrada. Na ventilação limitada por volume, o VC administrado a cada inspiração gera uma certa pressão na via aérea (que é medida e controlada nos ventiladores atuais). Da mesma forma, na ventilação limitada à pressão, a aplicação de um gradiente de pressão entre o ventilador e a via aérea resulta na entrada de um certo $\mathrm{VC}$, que pode ser medido e controlado. Independente do modo utilizado, é importante enfatizar que nenhum modo de ventilação convencional se mostra clinicamente superior ao outro no manejo de pacientes com SDRA, desde que respeitados os princípios de ventilação protetora.

Considerando que o controle preciso do VC é um fator muito importante no suporte da SDRA, modos de ventilação ciclados a tempo e limitados por volume são preferidos pela maioria dos intensivistas hoje em dia. Na ventilação ciclada a tempo e limitada por volume (controlada, assistida-controlada, mandatória intermitente ou mandatória intermitente com suporte de pressão), o operador determina o VC exato a ser administrado a cada ciclo mandatório do ventilador. As medições de pressão geradas por este volume ao final da inspiração (dinâmica), ou após uma pausa (pressão estática ou de plateau) são indicadores da complacência pulmonar na SDRA. Um pico de pressão inspiratório que aumenta ao longo do tempo para um determinado volume geralmente indica piora da complacência. De maneira análoga, uma diminuição do pico de pressão inspiratória geralmente indica uma melhora da complacência. Ventilação limitada por volume apresenta, tradicionalmente, uma onda de pressão de formato triangular, em contraste com a onda retangular da ventilação limitada à pressão (Figura 4). Como a área sob a curva de pressão reflete a pressão média de via aérea, modos limitados por volume 


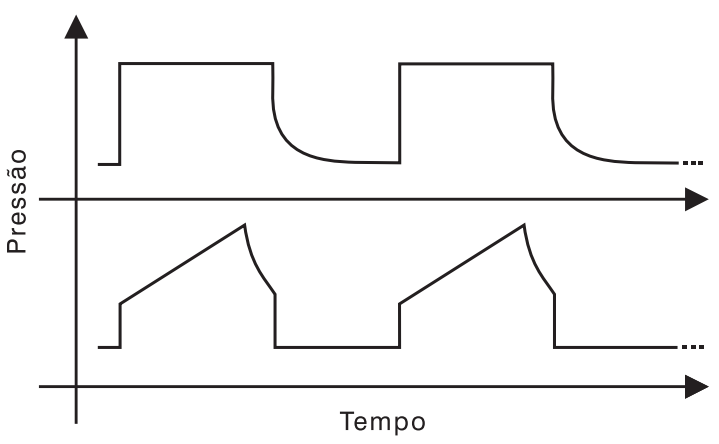

Figura 4 - Comparação do formato da onda de pressão dinâmica de vias aéreas durante ventilação com pressão controlada (a) e volume controlado (b)

(curva triangular) geralmente têm uma pressão média de vias aéreas ligeiramente menor que modos limitados à pressão (curva retangular). Entretanto, ventiladores modernos, como o Servo 300, oferecem o modo chamado de pressão regulada com volume controlado (PRVC), onde o formato de onda de pressão neste modo, por volume, se assemelha ao formato retangular de um modo por pressão. Assim sendo, o uso da PRVC tem conquistado boa aceitação no manejo de pacientes pediátricos com SDRA.

\section{Administração criteriosa de fluidos}

No pacientes com SDRA, o intensivista deve ponderar quanto à qualidade e quantidade dos fluidos a serem administrados. Para a expansão intravascular rápida, a decisão entre a administração de colóides ou cristalóides depende, de certa forma, mais da convicção pessoal do intensivista do que de ciência propriamente dita. Os que preferem administrar colóides justificam esta prática pelo fato de que estas substâncias são capazes de produzir uma expansão intravascular maior por unidade de volume administrado, permanecem mais tempo no espaço intravascular e aumentam a pressão coloidosmótica. Os que preconizam a administração de cristalóides o fazem por ser o cristalóide mais barato, mais prontamente acessível, por ser capaz de promover expansão intravascular equivalente aos colóides (quando volumes infundidos são ajustados) e por não induzirem ao risco de aumentar a pressão oncótica intersticial no pulmão, no caso de extravasamento capilar, como pode acontecer com os colóides. Estudos clínicos controlados não são conclusivos quanto à superioridade de colóides ou cristalóides. Portanto, a escolha do tipo de líquido para expansão intravascular rápida deve ser baseada nas necessidades do paciente em um determinado momento, considerando o tipo de perda ocorrida, a urgência da reanimação e a disponibilidade de fluidos, bem como a pressão coloidosmótica plasmática.
A quantidade de administração de fluidos em pacientes com SDRA também é alvo de debate. É inquestionável que pacientes com choque ou hipovolemia severa, ambos fatores de risco para SDRA, devem ser agressivamente ressuscitados, geralmente com volumes infundidos excedendo 60 $\mathrm{ml} / \mathrm{kg}$ na primeira hora, pois esta prática diminui a mortalidade e não está associada com aumento da incidência de SDRA $^{25}$. Tendo estabilizado hemodinamicamente o paciente com SDRA, o intensivista deve concentrar seus esforços em minimizar o extravasamento capilar e edema pulmonar que ocorre na SDRA. Estudos em animais com lesão pulmonar aguda indicam que o acúmulo de líquidos no pulmão pode ser atenuado quando se reduz a pressão do átrio esquerdo ${ }^{26}$. Esta estratégia de limitar a administração de fluidos também tem suporte em alguns estudos clínicos em pacientes com SDRA ${ }^{27,28}$. O grupo de estudos norteamericano, que envolve 24 hospitais (ARDS Network) organizados para o estudo de SDRA, está atualmente conduzindo um estudo multicêntrico, randomizado e controlado da administração de fluidos de forma "conservadora", em comparação com administração "liberal". Enquanto os resultados deste estudo ainda não estão disponíveis, uma recomendação sensata é a de manter o volume intravascular no nível mais baixo que permita a manutenção da perfusão sistêmica adequada, avaliada pela função renal, cardíaca e pelo estado do equilíbrio ácido-base.

\section{Ventilação não convencional}

\section{Ventilação de alta freqüência (VAF)}

Modalidades de ventilação mecânica que empregam freqüências suprafisiológicas, geralmente entre 60 e 900 ciclos por minuto, são conhecidas coletivamente como VAF. Vários tipos de VAF são disponíveis, apesar de que apenas a ventilação com pressão positiva de alta freqüência (VPPAF), ventilação a jato (VJAF) e ventilação oscilatória de alta freqüência (VOAF) têm significativa penetração na prática clínica. Estudos clínicos com VPPAF e VJAF em comparação com ventilação convencional foram desapontadores e resultaram no virtual abandono dessas modalidades no manejo de pacientes com SDRA ${ }^{29}$. O uso da VOAF, entretanto, tem forte suporte em estudos de modelos experimentais de $\operatorname{SDRA}^{17,30,31}$ e suficiente evidência clínica para justificar seu uso em situações selecionadas ${ }^{32-34}$.

$\mathrm{Na}$ VOAF, volumes correntes que se aproximam ao espaço morto são colocados e retirados ativamente da via aérea com uma freqüência entre 3 e 15 hertz (180 a 900 ciclos por minuto) através do movimento de um pistão ou de um diafragma. A proposta vantagem da VOAF é que, devido aos minúsculos volumes correntes em cada ciclo, esta modalidade é capaz de ventilar pacientes com SDRA em uma "zona de segurança" que evita tanto a hiperinsuflação alveolar na inspiração quanto o fechamento e reabertura cíclica de alvéolos na expiração (Figura 2). A oxigenação e a ventilação são controladas independentemente na VOAF. $\mathrm{O}$ controle da pressão média de vias aéreas determina o 
estado de insuflação pulmonar e, conseqüentemente, a oxigenação. O controle da amplitude de oscilação determina indiretamente o volume corrente de cada ciclo respiratório e, conseqüentemente, a eficiência da ventilação (eliminação de $\mathrm{CO}_{2}$ ). Assim sendo, a VOAF é ideal em situações nas quais o paciente com SDRA tem piora da complacência pulmonar com hipoxemia, resultando em necessidade de diminuição do VC na ventilação convencional para evitar pico de pressão inspiratória elevado e resultando em acidose respiratória importante. A constatação de que a VOAF pode favoravelmente influenciar o ambiente inflamatório pulmonar em modelos experimentais ${ }^{17,31,35}$, bem como reduzir a incidência de doença pulmonar crônica ${ }^{32,34}$, é responsável pelo entusiasmo para com esta modalidade e pelo seu uso cada vez mais precoce em pacientes com SDRA. O uso de VOAF no paciente pediátrico com SDRA exige sedação profunda e relaxamento neuromuscular, uma vez que movimentos inspiratórios espontâneos interferem com a mecânica do movimento de gás nesta modalidade.

\section{Ventilação não invasiva}

A aplicação de pressão positiva de forma não invasiva (CPAP ou BiPAP) em pacientes com SDRA é capaz de atenuar, mas apenas temporariamente, a redução de capacidade residual funcional responsável pela hipoxemia progressiva que é característica desta patologia. $\mathrm{O}$ uso de CPAP causa uma transitória melhora de oxigenação, mas não está associado com redução da necessidade de intubação, estadia hospitalar ou mortalidade em pacientes com SDRA ${ }^{10}$. O uso de CPAP na SDRA está ainda associado a uma maior incidência de efeitos adversos ${ }^{10}$. Assim sendo, o uso de CPAP na profilaxia e no tratamento de pacientes com SDRA não é recomendado.

\section{Ventilação líquida parcial}

A ventilação líquida parcial (VLP) é uma técnica que emprega substâncias perfluoronadas de carbono que dispõe de grande capacidade de dissolução de oxigênio e gás carbônico. Na VLP, o perfluorocarbono líquido é colocado no pulmão por via endotraqueal, de forma a ocupar a capacidade residual funcional, enquanto que volumes de gás são introduzidos através de um ventilador convencional a cada ciclo inspiratório ${ }^{36}$. A vantagem potencial da VLP na SDRA vem do fato de que, quando o pulmão é ocupado por líquido, ele passa a ter uma tensão superficial uniforme, ao contrário da tensão superficial heterogênea característica da SDRA. Isto ocorre porque o perfluorocarbono forma uma interface líquido-líquido na superfície alveolar, em contraste com a interface gás-líquido encontrada na ventilação convencional. Um perfluorocarbono de grau de pureza médica chamado perflubron (C8-F17-Br1) tem sido testado com sucesso no tratamento da lesão pulmonar aguda, em laboratório. Hoje sabemos que o perflubron, assim como outros perfluorocarbonos que eram considerados biolo- gicamente inertes, têm efeitos biológicos antiinflamatórios e protegem componentes celulares contra dano oxidativo ${ }^{37-41}$. Entretanto, o entusiasmo pela VLP em laboratório não tem correspondido na arena clínica. Estudos controlados em crianças e adultos com SDRA e lesão pulmonar aguda não demonstraram superioridade da VLP, em comparação com a ventilação protetora convencional ${ }^{42}$. Novos estudos ainda são necessários para testar o impacto desta modalidade em situações clínicas específicas, como recrutamento pulmonar progressivo (PEEP líquido) e administração intrapulmonar de drogas ou vetores virais para terapia genética. No presente momento, esta modalidade não é disponível para uso fora do ambiente experimental, e não pode ser recomendada no tratamento da SDRA.

\section{Terapia medicamentosa}

Reposição de surfactante

$\mathrm{O}$ sucesso da terapia com surfactante em neonatos prematuros, associado ao fato de que o sistema surfactante é disfuncional em pacientes com SDRA, levou intensivistas a especular o possível papel dessa substância no tratamento desta patologia. Entretanto, o uso de surfactante em pacientes adultos não se mostrou efetivo em melhorar a oxigenação, encurtar a duração da ventilação mecânica, ou reduzir mortalidade em um estudo clínico controlado ${ }^{43}$. Possíveis explicações para tal incluem o método de administração empregado (aerossol), que resulta em retenção de menos de $5 \%$ da dose, bem como o tipo de surfactante utilizado (preparação fosfolipídica sem proteínas de surfactante). Novas preparações de surfactante extraídos de pulmões bovinos que contêm fosfolipídios, lipídios neutros e proteínas hidrofóbicas de surfactante tipo B e C são consideradas mais eficazes e estão sendo testadas em pacientes com SDRA, por administração através do tubo endotraqueal ${ }^{44}$. Até que estudos definitivos estejam disponíveis, o uso rotineiro de surfactante em pacientes com SDRA não pode ser recomendado, sendo reservado para uso de exceção em situações especiais, nas quais o recrutamento de segmentos pulmonares não pode ser atingida com métodos mais convencionais. Mesmo nestas situações, a utilidade do surfactante na SDRA é questionável.

\section{Óxido nítrico}

O óxido nítrico é um potente vasodilatador que pode ser administrado por via inalatória, causando um efeito de relaxamento vascular pulmonar. O óxido nítrico administrado por via inalatória chega até ao alvéolo, onde entra em contato direto com a vasculatura pulmonar. Durante sua migração através da parede do vaso sangüíneo, o óxido nítrico causa um relaxamento direto da camada muscular, antes de alcançar o lúmen vascular. $\mathrm{O}$ óxido nítrico é então rapidamente desativado por ligação à hemoglobina, resultando na formação de metemoglobina e impedindo o indesejável efeito de vasodilatação 
sistêmica. $\mathrm{O}$ efeito vasodilatador pulmonar do óxido nítrico, associado ao fato de que a vasculatura alvo é aquela adjacente a áreas ventiladas do pulmão, faz com que o efeito resultante seja não apenas o de uma redução da resistência vascular pulmonar, mas também uma diminuição do desequilíbrio entre ventilação e perfusão, e uma melhora da oxigenação. $O$ uso de óxido nítrico em neonatos com hipertensão pulmonar tem alcançado grande sucesso, com diminuição de morbidade e redução da necessidade de suporte extracorpóreo ${ }^{45}$. Entretanto, seu uso em pacientes com SDRA tem sido desapontador. Apesar de proporcionar uma melhora transitória de oxigenação, este benefício tem curta duração e não se traduz em ganho objetivo. O uso do óxido nítrico na SDRA não reduz a mortalidade, ou a duração da ventilação mecâni$\mathrm{ca}^{46} \mathrm{e}$, portanto, não pode ser recomendado rotineiramente na prática clínica. O óxido nítrico pode ser usado como terapia de exceção no resgate temporário de pacientes com hipoxemia refratária a intervenções mais convencionais.

\section{Corticosteróides}

O fato de que o dano pulmonar agudo na SDRA é primariamente resultante de um agressivo processo inflamatório fez com que intensivistas considerassem drogas antiinflamatórias, em geral, e os corticosteróides, em particular, como alternativas terapêuticas lógicas. Entretanto, o uso de corticosteróides não previne o aparecimento de $\mathrm{SDRA}^{47}$, nem apresenta benefícios, quando empregados na fase inicial do quadro clínico ${ }^{48}$. Corticosteróides parecem ter alguma utilidade quando iniciados na fase mais tardia da doença, marcada pela reorganização do infiltrado inflamatório agudo e alveolite fibrosante ${ }^{49}$. Atualmente está em andamento um estudo multicêntrico randomizado e controlado, norte-americano (ARDS Network), avaliando a eficácia do tratamento de altas doses de metilprednisolona na fase tardia da SDRA. Entretanto, devido ao fato de que o tratamento com altas doses de corticosteróides pode elevar o risco de infecções secundárias e outros efeitos adversos, o uso de rotina desta terapia na SDRA ainda não pode ser recomendado. Na nossa prática clínica, reservamos o uso de corticosteróides como terapia de resgate no paciente com SDRA severa na fase tardia da doença (terceira ou quarta semana), que não apresenta progresso na diminuição do suporte ventilatório empregado.

\section{Outros agentes moduladores de inflamação}

Apesar de terem apresentado resultados promissores em modelos experimentais de lesão pulmonar aguda, o benefício de drogas não esteróides com efeito antiinflamatório como a indometacina, o ibuprofeno, a procisteína, a lisofilina e o cetoconazol não foi demonstrado na arena clínica ${ }^{50}$. O uso dessas drogas no tratamento de pacientes com SDRA, portanto, não pode ser recomendado.

\section{Oxigenação de membrana extracorpórea (OMEC)}

A OMEC consiste no uso de um complexo circuito de cânulas vasculares, tubos, bombas, oxigenador, aquecedor e sistema de monitorização, usado para propiciar suporte respiratório (no caso de OMEC veno-venoso) ou cardiorrespiratório (no caso de OMEC venoarterial). Até hoje, cerca de 25.000 pacientes foram submetidos à OMEC em todo o mundo, com uma sobrevida geral em torno de $75 \%$. A maioria destes pacientes (17.000) são neonatos com hipertensão pulmonar refratária, sendo que a experiência pediátrica e adulta com OMEC para tratamento de SDRA se limita a cerca de 3.000 casos (comunicação pessoal, ECMO Registry of the Extracorporeal Life Support Organization (ELSO), Ann Arbor, Michigan, novembro, 2002). A OMEC reduz a mortalidade de neonatos com hipertensão pulmonar persistente secundária à aspiração de mecônio (sobrevida de 94\%), mas mostra resultados mais modestos em crianças maiores com SDRA (sobrevida de 52\%). Estudos clínicos do uso de OMEC, ou de um sistema extracorpóreo de eliminação de dióxido de carbono em adultos com SDRA, não mostrou benefícios na redução da mortalidade ${ }^{51}$. Entretanto, os resultados da terapia com OMEC no registro internacional de suporte extracorpóreo em pacientes pediátricos com SDRA refratária a todas as formas de tratamento, bem como na nossa prática pessoal, sugerem fortemente o valor desta técnica em casos selecionados.

A indicação de OMEC na SDRA se restringe a pacientes com o grau mais severo de falência pulmonar aguda, sendo potencialmente reversível, mas não responsivo aos tratamentos convencional e não convencional menos invasivos. A premissa básica para indicação da OMEC é que a morte do paciente seja presumivelmente iminente sem o uso desta tecnologia. Nos últimos 7 anos, 22 destes pacientes pediátricos com falência respiratória extrema foram submetidos à OMEC para tratamento da SDRA no Children's Hospital of Buffalo, com uma sobrevida de 54\%. Esses resultados são compatíveis com os de outros centros de excelência na América do Norte e são o grande impedimento para a realização de estudos randomizados. Como nós, a maioria dos centros que praticam essa modalidade consideram uma violação ética alocar pacientes candidatos à OMEC a um grupo controle sem intervenção, uma vez que estes pacientes têm uma mortalidade projetada em torno de $100 \%$.

\section{Terapia de posição}

A simplicidade e o baixo custo do uso da posição prona, associados a relatos de melhora da oxigenação em 60 a $70 \%$ dos pacientes com SDRA, popularizou o uso dessa modalidade terapêutica. Vários mecanismos têm sido propostos para explicar esse efeito no paciente em posição prona, como melhora da relação entre ventilação e perfusão $0^{52}$, aumento do volume pulmonar ao final da expiração ${ }^{53} \mathrm{e}$ mudanças regionais de ventilação associadas a alterações mecânicas da parede torácica ${ }^{54}$. Entretanto, como já foi demonstrado anteriormente, melhoras na oxigenação não 
necessariamente se traduzem em redução de mortalidade na SDRA $^{15}$. Recentemente, Gattinoni e colaboradores ${ }^{55}$ reportaram os resultados de um estudo multicêntrico e controlado, no qual pacientes com SDRA foram randomizados a receber tratamento convencional (posição supina) ou tratamento na posição prona, por 6 ou mais horas por dia, por 10 dias. Nesse estudo, apesar de causar uma melhora na oxigenação, o uso da posição prona não resultou em uma redução de mortalidade ${ }^{55}$. Várias teorias podem explicar esses achados. A mais simples é que o uso da posição prona não previne ou atenua o avanço da lesão pulmonar em pacientes com SDRA. Por outro lado, apesar de incluir 304 pacientes, esse estudo provavelmente não tinha poder estatístico adequado para mostrar diferenças entre os grupos, uma vez que SDRA em estudos clínicos mostra características heterogêneas, exigindo amostras de grande tamanho. Os pacientes randomizados ao grupo de posição prona assumiram essa posição por cerca de 7 horas por dia (ou apenas 30\% do tempo), e por um máximo de 10 dias. É possível que o tempo de exposição limitado à posição prona possa explicar a falha dessa estratégia.

Um estudo multicêntrico em pacientes pediátricos com SDRA envolvendo o uso da posição prona durante a maior parte do dia, e até a resolução da falência respiratória, está em andamento em UTIs terciárias na América do Norte. Até que resultados concretos estejam disponíveis, a recomendação de colocar pacientes com SDRA na posição prona, na tentativa de melhorar a oxigenação e permitir exposição a menores concentrações de oxigênio, parece ter razoável embasamento teórico e poucos riscos ou custos associados.

\section{Prevenção e diagnóstico precoce de infecções inter- correntes}

Por requerer tecnologia invasiva por períodos prolongados, como cateteres vasculares e vesicais, intubação endotraqueal e ventilação mecânica, o paciente com SDRA é alvo frequiente de infecções secundárias, principalmente as pulmonares. O diagnóstico precoce e o tratamento preciso dessas infecções são de extrema importância, uma vez que pneumonias secundárias agem como insulto pró-inflamatório adicional na SDRA. O diagnóstico radiológico de infecções pulmonares secundárias em pacientes com SDRA é complicado pelo fato de que esses pacientes apresentam anormalidades radiológicas pré-existentes. O diagnóstico clínico também apresenta desafios, uma vez que sinais como febre, leucocitose e aumento de secreções traqueais podem fazer parte da patologia de base. Na prática clínica, o diagnóstico precoce pode ser feito através da integração de alterações radiológicas, aspecto e celularidade das secreções traqueais e exames culturais de rotina (aspirado traqueal, lavado broncoalveolar e hemocultura).

Assim como nas demais infecções hospitalares, a prevenção é a melhor maneira de reduzir o risco de infecções pulmonares secundárias. O isolamento de pacientes imunodeprimidos ou contagiosos e o uso de precauções universais de contato, bem como a lavagem freqüente de mãos, são manobras simples e altamente efetivas. O uso criterioso de antibioticoterapia, baseado no antibiograma de organismos isolados por cultura ou em dados epidemiológicos locais, também tem papel importante na prevenção de infecções secundárias.

\section{Analgesia e sedação}

O conforto do paciente com SDRA durante a internação em UTI deve ocupar papel de destaque na estratégia terapêutica. Pacientes na fase aguda da doença devem receber infusões de medicamentos para aliviar o estresse emocional e o desconforto físico inerente à patologia, bem como em antecipação a procedimentos dolorosos. Nossa prática é de manter pacientes com SDRA sob sedação e analgesia contínuas, associada à reavaliação diária dessas necessidades. Infusões de midazolam $(0,1 \mathrm{mg} / \mathrm{kg} / \mathrm{h})$ e fentanil $(2 \mu \mathrm{g} / \mathrm{kg} / \mathrm{h})$ são utilizadas na maioria dos pacientes com ajuste das doses conforme a necessidade clínica, não sendo infreqüente doses 10 vezes mais altas do que as originais, quando da terceira semana da evolução clínica. Pacientes submetidos à hipercapnia permissiva e VOAF necessitam de infusão de bloqueadores neuromusculares, como o vecurônio $(0,1 \mathrm{mg} / \mathrm{kg} / \mathrm{h})$. Pacientes com mecânica pulmonar bastante comprometida e na fase aguda da doença também frequientemente necessitam de bloqueadores neuromusculares.

\section{Suporte nutricional}

Pacientes com SDRA apresentam necessidades calóricas diárias elevadas em função do estresse do trauma, sepse, cirurgia ou do processo inflamatório que acompanham a lesão pulmonar da SDRA. Esses pacientes necessitam de pronta instituição de nutrição parenteral ou enteral, uma vez que o déficit calórico pode resultar em alterações dos mecanismos de defesa, bem como da capacidade de cicatrização pulmonar. Nossa preferência é pela alimentação enteral contínua por via nasoduodenal, assim que tecnicamente possível. Nutrição parenteral total deve ser iniciada imediatamente em pacientes que demonstram intolerância ou contra-indicação à alimentação enteral. Entre as complicações da nutrição parenteral, deve-se ressaltar a hipercapnia, que pode ser observada quando há aporte excessivo de carboidratos, alterando o quociente respiratório.

\section{Suporte psicológico}

As necessidades psicossociais da família e do paciente com SDRA são bastante complexas. Mesmo em pacientes adequadamente sedados, fatores como a ansiedade em relação à incerteza do desfecho clínico, a impossibilidade de vocalizar devido à via aérea artificial, a ocasional dor decorrente de procedimentos invasivos e a alteração dos ritmos de sono e de vigília, entre outros, não podem ser negligenciados pela equipe médica. Atenção deve ser dis- 
pensada para que se explique ao paciente (sempre que possível) e à família todos os procedimentos diagnósticos e terapêuticos, assim como o curso natural e prognóstico da patologia. É comum para pacientes adolescentes e crianças maiores, em fase de recuperação da SDRA, a apresentação de delírio, depressão ou alterações de padrões circadianos durante prolongadas hospitalizações em ambiente de UTI. Tais manisfestações requerem, freqüentemente, a participação de consultor da área de psiquiatria para seguimento de pacientes durante a reabilitação e após a alta hospitalar. A equipe médica multidisciplinar deve estar sempre atenta e disponível às necessidades psicológicas do paciente com SDRA e de sua família, principalmente porque internações em UTI devido à SDRA severa são prolongadas e, geralmente, marcadas pela alternância entre momentos de frustração e de otimismo.

\section{Monitorização do paciente}

O paciente com SDRA representa um estrato relativamente grave da população de uma UTI terciária. Assim sendo, esses pacientes requerem um nível de monitorização alto para que os dados sejam obtidos e integrados em tempo real na individualização da estratégia de tratamento. Pacientes com SDRA requerem, rotineiramente, a colocação de cateter arterial para a monitorização contínua de pressão arterial, bem como para a obtenção de gasometrias arteriais seriadas. Um cateter venoso central, dotado de 2 a 3 lúmens, é empregado para administração de fluidos e medicamentos, assim como para a medição contínua da pressão venosa central. Uma sonda vesical permite a medição precisa das perdas urinárias e o controle do balanço hídrico. A oximetria de pulso contínua é usada para avaliação da oxigenação em tempo real. A análise das curvas de dióxido de carbono exalado proporciona uma inferência contínua da ventilação, perfusão pulmonar e espaço morto. A monitorização respiratória, por meio de interface gráfica, permite uma visualização em tempo real de uma série de parâmetros respiratórios derivados de pressão, fluxo, tempo e volume. A ecocardiografia seriada é uma boa maneira de monitorar-se o grau de enchimento atrial (pré-carga), assim como a função cardíaca resultante de diferentes combinações de drogas inotrópicas e estados de expansão intravascular. Na nossa experiência, o cateter de artéria pulmonar (Swan-Ganz) tem pouca utilidade no paciente com SDRA sem comprometimento cardíaco de base. $\mathrm{O}$ uso desses cateteres raramente altera o manejo baseado em dados obtidos com a tecnologia auxiliar descrita acima. Pacientes submetidos a bloqueio neuromuscular contínuo devem ser monitorados com eletroestimuladores, para evitar uso de doses exageradas.

\section{Referências bibliográficas}

1. Bernard GR, Artigas A, Brigham KL, Carlet J, Falke K, Hudson $\mathrm{L}$, et al. The American-European Consensus Conference on ARDS. Definitions, mechanisms, relevant outcomes, and clinical trial coordination. Am J Respir Crit Care Med 1994;149:818-24.

2. Luhr OR, Antonsen K, Karlsson M, Aardal S, Thorsteinsson A, Frostell CG, et al. Incidence and mortality after acute respiratory failure and acute respiratory distress syndrome in Sweden, Denmark, and Iceland. The ARF Study Group. Am J Respir Crit Care Med 1999;159:1849-61.

3. Suchyta MR, Clemmer TP, Orme JF Jr, Morris AH, Elliott CG. Increased survival of ARDS patients with severe hypoxemia (ECMO criteria). Chest 1991;99:951-5.

4. Milberg JA, Davis DR, Steinberg KP, Hudson LD. Improved survival of patients with acute respiratory distress syndrome (ARDS): 1983-1993. JAMA 1995;273:306-9.

5. Amato MB, Barbas CS, Medeiros DM, Magaldi RB, Schettino GP, Lorenzi-Filho G, et al. Effect of a protective-ventilation strategy on mortality in the acute respiratory distress syndrome. N Engl J Med 1998;338:347-54.

6. Ashbaugh DG, Bigelow DB, Petty TL, Levine BE. Acute respiratory distress in adults. Lancet 1967;2:319-23.

7. Matalon S, Holm BA, Loewen GM, Baker RR, Notter RH. Sublethal hyperoxic injury to the alveolar epithelium and the pulmonary surfactant system. Exp Lung Res 1988;14 Suppl: 1021-33.

8. Nader-Djalal N, Knight PR 3rd, Thusu K, Davidson BA, Holm BA, Johnson KJ, et al. Reactive oxygen species contribute to oxygen-related lung injury after acid aspiration. Anesth Analg 1998;87:127-33.

9. Yusa T, Crapo JD, Freeman BA. Hyperoxia enhances lung and liver nuclear superoxide generation. Biochim Biophys Acta 1984;798:167-74.

10. Delclaux C, L'Her E, Alberti C, Mancebo J, Abroug F, Conti G, et al. Treatment of acute hypoxemic nonhypercapnic respiratory insufficiency with continuous positive airway pressure delivered by a face mask: A randomized controlled trial. JAMA 2000;284:2352-60.

11. Gattinoni L, Presenti A, Torresin A, Baglioni S, Rivolta M, Rossi $\mathrm{F}$, et al. Adult respiratory distress syndrome profiles by computed tomography. J Thorac Imaging 1986;1:25-30.

12. Gattinoni L, Pesenti A, Bombino M, Baglioni S, Rivolta M, Rossi F, et al. Relationships between lung computed tomographic density, gas exchange, and PEEP in acute respiratory failure. Anesthesiology 1988;69:824-32.

13. Slutsky AS. Lung injury caused by mechanical ventilation. Chest. 1999;116(1 Suppl):9-15.

14. Ranieri VM, Suter PM, Tortorella C, De Tullio R, Dayer JM, Brienza A, et al. Effect of mechanical ventilation on inflammatory mediators in patients with acute respiratory distress syndrome: a randomized controlled trial. JAMA 1999;282:54-61.

15. The Acute Respiratory Distress Syndrome Network. Ventilation with lower tidal volumes as compared with traditional tidal volumes for acute lung injury and the acute respiratory distress syndrome. N Engl J Med 2000;342:1301-8.

16. Dreyfuss D, Soler P, Basset G, Saumon G. High inflation pressure pulmonary edema. Respective effects of high airway pressure, high tidal volume, and positive end-expiratory pressure. Am Rev Respir Dis 1988;137:1159-64.

17. Rotta AT, Gunnarsson B, Fuhrman BP, Hernan LJ, Steinhorn DM. Comparison of lung protective ventilation strategies in a rabbit model of acute lung injury. Crit Care Med 2001;29:2176-84. 
18. Brochard L, Roudot-Thoraval F, Roupie E, Delclaux C, Chastre J, Fernandez-Mondejar E, et al. Tidal volume reduction for prevention of ventilator-induced lung injury in acute respiratory distress syndrome. The Multicenter Trial Group on Tidal Volume reduction in ARDS. Am J Respir Crit Care Med 1998;158:1831-8.

19. Stewart TE, Meade MO, Cook DJ, Granton JT, Hodder RV, Lapinsky SE, et al. Evaluation of a ventilation strategy to prevent barotrauma in patients at high risk for acute respiratory distress syndrome. Pressure- and Volume-Limited Ventilation Strategy Group. N Engl J Med 1998;338:355-61.

20. Ranieri VM, Giunta F, Suter PM, Slutsky AS. Mechanical ventilation as a mediator of multisystem organ failure in acute respiratory distress syndrome. JAMA 2000;284:43-4.

21. Tremblay L, Valenza F, Ribeiro SP, Li J, Slutsky AS. Injurious ventilatory strategies increase cytokines and c-fos m-RNA expression in an isolated rat lung model. J Clin Invest 1997;99: 944-52.

22. The Acute Respiratory Distress Syndrome Network. Prospective, Randomized, Multi-Center Trial of Higher End-expiratory Lung Volume/Lower FiO2 versus Lower End-expiratory Lung Volume/ Higher FiO2 Ventilation in Acute Lung Injury and Acute Respiratory Distress Syndrome [site na internet] Disponível: http://hedwig.mgh.harvard.edu/ardsnet/ards04.html. Acessado: 7 de abril de 2003 .

23. Carvalho CR, Barbas CS, Medeiros DM, Magaldi RB, Lorenzi Filho G, Kairalla RA, et al. Temporal hemodynamic effects of permissive hypercapnia associated with ideal PEEP in ARDS. Am J Respir Crit Care Med 1997;156:1458-66.

24. Feihl F, Perret C. Permissive hypercapnia. How permissive should we be? Am J Respir Crit Care Med 1994;150:1722-37.

25. Carcillo JA, Davis AL, Zaritsky A. Role of early fluid resuscitation in pediatric septic shock. JAMA 1991;266:1242-5.

26. Prewitt RM, McCarthy J, Wood LD. Treatment of acute low pressure pulmonary edema in dogs: relative effects of hydrostatic and oncotic pressure, nitroprusside, and positive end-expiratory pressure. J Clin Invest 1981;67:409-18.

27. Mitchell JP, Schuller D, Calandrino FS, Schuster DP. Improved outcome based on fluid management in critically ill patients requiring pulmonary artery catheterization. Am Rev Respir Dis 1992;145:990-8.

28. Humphrey H, Hall J, Sznajder I, Silverstein M, Wood L. Improved survival in ARDS patients associated with a reduction in pulmonary capillary wedge pressure. Chest 1990;97:1176-80.

29. Wiswell TE, Graziani LJ, Kornhauser MS, Cullen J, Merton DA, McKee L, et al. High-frequency jet ventilation in the early management of respiratory distress syndrome is associated with a greater risk for adverse outcomes. Pediatrics 1996;98:1035-43.

30. Froese AB. Role of lung volume in lung injury: HFO in the atelectasis-prone lung. Acta Anaesthesiol Scand Suppl 1989;90:126-30.

31. McCulloch PR, Forkert PG, Froese AB. Lung volume maintenance prevents lung injury during high frequency oscillatory ventilation in surfactant-deficient rabbits. Am Rev Respir Dis 1988; 137:1185-92.

32. Arnold JH, Hanson JH, Toro-Figuero LO, Gutierrez J, Berens RJ, Anglin DL. Prospective, randomized comparison of highfrequency oscillatory ventilation and conventional mechanical ventilation in pediatric respiratory failure. Crit Care Med 1994;22:1530-9.

33. Derdak S, Mehta S, Stewart TE, Smith T, Rogers M, Buchman TG, et al. High-frequency oscillatory ventilation for acute respiratory distress syndrome in adults: a randomized, controlled trial. Am J Respir Crit Care Med 2002;166:801-8.
34. Courtney SE, Durand DJ, Asselin JM, Hudak ML, Aschner JL, Shoemaker CT. High-frequency oscillatory ventilation versus conventional mechanical ventilation for very-low-birth-weight infants. N Engl J Med 2002;347:643-52.

35. Sugiura M, McCulloch PR, Wren S, Dawson RH, Froese AB. Ventilator pattern influences neutrophil influx and activation in atelectasis-prone rabbit lung. J Appl Physiol 1994;77:1355-65.

36. Fuhrman BP, Paczan PR, DeFrancisis M. Perfluorocarbonassociated gas exchange. Crit Care Med 1991;19:712-22.

37. Rotta AT, Steinhorn DM. Partial liquid ventilation reduces pulmonary neutrophil accumulation in an experimental model of systemic endotoxemia and acute lung injury. Crit Care Med 1998;26:1707-15.

38. Rotta AT, Gunnarsson B, Hernan LJ, Fuhrman BP, Steinhorn DM. Partial liquid ventilation with perflubron attenuates in vivo oxidative damage to proteins and lipids. Crit Care Med 2000;28:202-8.

39. Rotta AT, Gunnarsson B, Hernan LJ, Fuhrman BP, Steinhorn DM. Partial liquid ventilation influences pulmonary histopathology in an animal model of acute lung injury. J Crit Care 1999;14:84-92.

40. Rotta AT, Gunnarsson B, Hernan LJ, Fuhrman BP, Steinhorn DM. Perflubron protects cell monolayers against direct in vitro oxidative injury. Crit Care Med 1999;27:A40.

41. Rotta AT, Gunnarsson B, Hernan LJ, Fuhrman BP, Steinhorn DM. Perfluorocarbon protects against fatty acid oxidation in a non-biological, in vitro model. Crit Care Med 1999;27:A133.

42. Hirschl RB, Croce M, Gore D, Wiedemann H, Davis K, Zwischenberger J, et al. Prospective, randomized, controlled pilot study of partial liquid ventilation in adult acute respiratory distress syndrome. Am J Respir Crit Care Med 2002;165:781-7.

43. Anzueto A, Baughman RP, Guntupalli KK, Weg JG, Wiedemann HP, Raventos AA, et al. Aerosolized surfactant in adults with sepsis-induced acute respiratory distress syndrome. Exosurf Acute Respiratory Distress Syndrome Sepsis Study Group. N Engl J Med 1996;334:1417-21.

44. Willson DF, Zaritsky A, Bauman LA, Dockery K, James RL, Conrad D, et al. Instillation of calf lung surfactant extract (calfactant) is beneficial in pediatric acute hypoxemic respiratory failure. Members of the Mid-Atlantic Pediatric Critical Care Network. Crit Care Med 1999;27:188-95.

45. Clark RH, Kueser TJ, Walker MW, Southgate WM, Huckaby JL, Perez JA, et al. Low-dose nitric oxide therapy for persistent pulmonary hypertension of the newborn. Clinical Inhaled Nitric Oxide Research Group. N Engl J Med 2000;342:469-74.

46. Dellinger RP, Zimmerman JL, Taylor RW, Straube RC, Hauser DL, Criner GJ, et al. Effects of inhaled nitric oxide in patients with acute respiratory distress syndrome: results of a randomized phase II trial. Inhaled Nitric Oxide in ARDS Study Group. Crit Care Med 1998;26:15-23.

47. Luce JM, Montgomery AB, Marks JD, Turner J, Metz CA, Murray JF. Ineffectiveness of high-dose methylprednisolone in preventing parenchymal lung injury and improving mortality in patients with septic shock. Am Rev Respir Dis 1988;138:62-8.

48. Bernard GR, Luce JM, Sprung CL, Rinaldo JE, Tate RM, Sibbald WJ, et al. High-dose corticosteroids in patients with the adult respiratory distress syndrome. N Engl J Med 1987;317:1565-70.

49. Meduri GU, Belenchia JM, Estes RJ, Wunderink RG, el Torky M, Leeper KV Jr. Fibroproliferative phase of ARDS. Clinical findings and effects of corticosteroids. Chest 1991;100:943-52.

50. ARDS N. Ketoconazole for early treatment of acute lung injury and acute respiratory distress syndrome: a randomized controlled trial. The ARDS Network. JAMA 2000;283:1995-2002. 
51. Morris AH, Wallace CJ, Menlove RL, Clemmer TP, Orme JF Jr, Weaver LK, et al. Randomized clinical trial of pressure-controlled inverse ratio ventilation and extracorporeal $\mathrm{CO} 2$ removal for adult respiratory distress syndrome. Am J Respir Crit Care Med 1994;149:295-305.

52. Pappert D, Rossaint R, Slama K, Gruning T, Falke KJ. Influence of positioning on ventilation-perfusion relationships in severe adult respiratory distress syndrome. Chest 1994;106:1511-6.

53. Douglas WW, Rehder K, Beynen FM, Sessler AD, Marsh HM. Improved oxygenation in patients with acute respiratory failure: the prone position. Am Rev Respir Dis 1977;115:559-66.

54. Pelosi P, Tubiolo D, Mascheroni D, Vicardi P, Crotti S, Valenza $\mathrm{F}$, et al. Effects of the prone position on respiratory mechanics and gas exchange during acute lung injury. Am J Respir Crit Care Med 1998;157:387-93.
55. Gattinoni L, Tognoni G, Pesenti A, Taccone P, Mascheroni D, Labarta $\mathrm{V}$, et al. Effect of prone positioning on the survival of patients with acute respiratory failure. N Engl J Med 2001; 345:568-73.

Endereço para correspondência:

Dr. Alexandre Tellechea Rotta

Division of Pediatric Critical Care

The Children's Hospital of Buffalo, 219

Bryant Street, Buffalo, NY 14222, USA

Tel.: (716) 878.7442 - Fax: (716) 878.7101

E-mail: arotta@buffalo.edu 